\title{
Ability of Non-Invasive Tear Break-Up Time to Determine Tear Instability in Contact Lens Wearers
}

Ersin Muhafiz ( $\nabla$ ersinmuhafiz@hotmail.com )

Kafkas Universitesi Tip Fakultesi https://orcid.org/0000-0002-8281-8767

Mehmet Siraç Demir

Van Training and Research Hospital: Van Egitim ve Arastirma Hastanesi

\section{Research Article}

Keywords: contact lenses, non-invasive tear break-up time (NIBUT)

Posted Date: February 22nd, 2021

DOI: https://doi.org/10.21203/rs.3.rs-190044/v1

License: (9) This work is licensed under a Creative Commons Attribution 4.0 International License. Read Full License

Version of Record: A version of this preprint was published at International Ophthalmology on October 22nd, 2021. See the published version at https://doi.org/10.1007/s10792-021-02079-3. 


\section{Abstract}

Purpose To evaluate the performance of precorneal and prelens non-invasive tear break-up time (NIBUT) measurements to determine tear instability in contact lens $(\mathrm{CL})$ wearers.

Methods In this study 50 eyes of CL wearers were evaluated. Precorneal first and average NIBUTs and fluorescein tear break-up time (FBUT) were measured before wearing CLs in the morning. Those with FBUT less than 10 seconds were considered to have tear instability. After wearing $C L$, first and average prelens NIBUTs were measured at the 30th minute. The receiver operating characteristic (ROC) curve was analyzed to evaluate the performance of NIBUT measurements in the diagnosis of tear instability.

Results The FBUT value had a significant correlation with first and average precorneal NIBUT values ( $p<$ $0.001, r=0.653$ and $p<0.001, r=0.628$,respectively). The FBUT value had no correlation with the prelens first and average NIBUT values ( $p=0.542$ and $p=0.263$,respectively). To understand the relationship between the precorneal and prelens NIBUT values measured by the automated method, their correlation was evaluated. There was no significant correlation between the precorneal and prelens NIBUTs (for all;p $>0.05$ ).

The area under curve (AUC) in ROC curve for the first and average precorneal NIBUTs were 0.842 $(p<0.001)$ and $0.810(p<0.001)$, respectively. The AUC values for the first and average prelens NIBUTs at the $30^{\text {th }}$ minute of $C L$ wear were 0.586 and 0.619 respectively ( $p=0.317$ and $p=0.166$, respectively).

Conclusions: Precorneal NIBUT measurements may be useful in diagnosing tear instability in CL wearers. Prelens NIBUT values are not yet capable of adequately defining the tear film dynamics.

\section{Introduction}

Since some changes occur in the ocular surface and tear functions due to the use of contact lens (CL), it is necessary to evaluate the ocular surface and tear stability to prevent CL-related complications [1, 2]. CLs separate the tear film into two layers, therefore they are considered to increase tear evaporation and cause dry eye $[3,4]$. Although CLs are very effective in correcting vision, nearly half of those who use CLs have dry eye symptoms by the end of the day due to tear instability, and some have to give up wearing CLs for this reason $[1,5,6]$. Failure to distribute the tear film evenly over the CL surface reduces contrast sensitivity and visual quality $[7,8]$. The evaluation of the quality of the precorneal and prelens tear film is, therefore, important in the follow-up of CL users, as well as guiding in the selection of lens material and determination of the wearing protocol.

Fluorescein tear break-up time (FBUT), which is the most commonly used method in determining tear film instability and expresses the time interval between a complete blink and the first dry spot in the precorneal tear film, is a standard clinical test for the diagnosis of tear dysfunction and evaluation of the effectiveness of related treatments $[9,10]$. Being simple and requiring no expensive equipment, FBUT measurements are widely used in clinical practice; however, this method cannot be used in CL users while 
they are wearing CLs on their eye since fluorescein needs to be applied. Another way to measure tear instability is non-invasive tear break-up time (NIBUT), which is an automated method based on the principle of evaluating irregularities in the tear by projecting Placido rings onto the precorneal tear film. The advantage of this method is that it can measure tear instability without the need for a foreign substance, such as fluorescein drops or strips; thus, it provides a practical solution for the evaluation of prelens tear instability in people wearing CLs $[11,12]$. Although the NIBUT method is used by eye care practitioners, there is not yet sufficient evidence of its ability to assess tear instability in CL wearers [11, 13].

It is important to measure prelens NIBUT since CL wear increases evaporation and dry eye symptoms may develop even in individuals with normal precorneal tear stability. This study aimed to evaluate the usability of precorneal and prelens NIBUT measurements with the Sirius multifunctional corneal topography device (Sirius, CSO, Florence, Italy) in the prediction and identification of tear instability in CL wearers. Thus, the ability of NIBUT to demonstrate precorneal or prelens tear film quality and whether it can be routinely used in CL users was investigated.

\section{Methods}

In this prospective study, 50 eyes of CL wearers who presented to our hospital for routine control were evaluated. The research was carried out in accordance with the principles of the Declaration of Helsinki after receiving informed consent of the patients. Approval was obtained from the Van Training and Research ethics committee to conduct the study. People with pterygium, keratoconus or corneal dystrophy, ocular allergy, history of eye surgery, refractive surgery or trauma, those with more than 0.75 diopters of keratometric astigmatism, and those using systemic drugs that could affect ocular surface or tear functions were excluded from the study.

\section{Study Protocol}

Firstly, refractive errors and keratometric values and visual acuity of the participants were determined. Then, detailed biomicroscopic anterior segment examination was performed. To ensure standardization in the study, a fresh $\mathrm{CL}$ with the same material and design was tested in all participants. The suitability of this newly tested CL (Acuvue Oasys, Johnson \& Johnson) was confirmed by evaluating the centralization and movement of the lens, and the visual acuity of the participant. Eligible participants were told not to use their own CLs for a week, and attend an examination at the hospital at 9 a.m. the following week. After one-week standardization, precorneal NIBUT and FBUT values were measured before wearing CL in the morning. After wearing CLs and waiting for 30 minutes to let the tear settle, the first and average prelens NIBUT values were measured over the CLs. NIBUT measurements were made by the same technician, and the FBUT measurements were undertaken by the same doctor. According to the internationally accepted limits (Dry Eye Workshop 2007 and 2017), FBUT values under 10 seconds are considered as tear instability $[11,14]$. For this reason, a baseline FBUT value of 10 seconds and above was considered as normal and less than 10 seconds as an indication of tear instability. The ability of 
NIBUT values to identify those with tear instability with respect to the baseline FBUT value obtained in the morning was investigated.

\section{Non-Invasive Tear Break-Up Time}

The NIBUT of the participants was measured in accordance with the manufacturer's instructions using Sirius multifunctional topography device. Just before starting the measurement, the image was full centered and sharpened. The patient is instructed to blink twice to spread the tear across the ocular surface, then keep the eyes open as much as possible. The device automatically starts measuring after the patient has blinked twice. The device evaluates time-dependent changes in the integrity of the tear film spread over the anterior corneal surface. The test is performed through videokeratoscopy, the video recording of the Placido disk ring projection on the patient's precorneal or prelens tear film. When the tear film is distributed homogeneously on the ocular surface, it causes a regular appearance in the rings, and any deformation or disruption in the tear film results in deformation in one or more rings at the measurement time and place. The system processes the video in real time and without user intervention. The first break up of tear film causes distortion or interruption in the Placido disc pattern, which is recorded as first NIBUT by the device. In the device, in order to assist and complement the diagnosis, after blinking, the average duration of all break-up intervals occurring in the precorneal or prelens tear film is recorded as average NIBUT. As an example, Fig. 1 shows the NIBUT maps of one of the participants in our study.

\section{Fluorescein Tear Break-Up Time}

A disposable fluorescein strip was applied over the inferior tear meniscus of the participants to measure the FBUT value. The participants were asked to blink several times for the homogeneous distribution of fluorescein on the ocular surface, and then to keep their eyes open as much as possible. The time elapsed from the blinking of the participants to the first black spot on the cornea was measured as the FBUT value using a cobalt blue filter on a biomicroscope. Those with a baseline FBUT of less than 10 seconds were considered to have tear instability, while 10 seconds or greater FBUT were considered to indicate a normal tear film $[10,11,14]$.

\section{Statistical Analysis}

Data obtained in the study were statistically analyzed using SPSS24 software (Statistical Package for the Social Sciences, IBM). Descriptive statistics were used for the variables of age, gender, and tear parameters. The normality of data distribution was evaluated by the Kolmogorov-Smirnov test. The relationship between FBUT and NIBUT (precorneal and prelens) was evaluated by Spearman correlation analysis since the data did not show normal distribution. Those with tear instability were determined based on internationally accepted cut-off values of basal FBUT measurements as mentioned earlier. Then, the receiver operator characteristic (ROC) curve analysis was conducted to measure the ability of first and average NIBUTs to identify tear instability [15]. The area under the curve (AUC), considered as an index of discriminating performance, assessed this ability. For first and average NIBUTs, the sensitivity, specificity and optimum cut-off value for predicting tear instability were determined using ROC curve analyses based on the highest possible Youden index $[16,17]$. Evaluations were made at the $95 \%$ 
confidence interval, and $\mathrm{p}$ values less than 0.05 were considered to indicate statistically significant differences.

\section{Results}

The average age of the participants was $22.16 \pm 4.83$ years (range 14-31 years). The mean precorneal first and average NIBUT values of individuals were $7.92 \pm 4.53 \mathrm{sec}$ and $9.41 \pm 3.67 \mathrm{sec}$, respectively prior to $\mathrm{CL}$ wear. The mean FBUT was $6.68 \pm 4.66 \mathrm{sec}$ before $\mathrm{CL}$ wear. Tear instability was observed in 32 $(64 \%)$ eyes based on FBUT value. At the 30th minute of CL wear, the prelens first and average NIBUT values were $4.77 \pm 4.16 \mathrm{sec}$, and $7.60 \pm 3.36 \mathrm{sec}$ respectively (Table 1 ).

Table 1

The tear break-up time values of participants.

\begin{tabular}{|ll|}
\hline Variable & Mean \pm SD (secs) \\
\hline Fluorescein Tear Break-up Time & $6.68 \pm 4.66$ \\
\hline Precorneal first NIBUT before CL insertion & $7.92 \pm 4.53$ \\
\hline Precorneal average NIBUT before CL insertion & $9.41 \pm 3.67$ \\
\hline Prelens first NIBUT after CL insertion & $4.77 \pm 4.16$ \\
\hline Prelens average NIBUT after CL insertion & $7.60 \pm 3.36$ \\
\hline CL: Contact Lens, NIBUT: Noninvasive Tear Break-Up Time, SD: Standard Deviation \\
\hline
\end{tabular}

When the correlation between the FBUT value and the precorneal and prelens NIBUT values are evaluated, a significant correlation was detected between the FBUT value and the precorneal first and average NIBUT values ( $p<0.001, r=0.653$ and $p<0.001, r=0.628$, respectively), but no significant correlation was found between the FBUT value and the prelens first and average NIBUT values $(p=0.542$ and $p=0.263$, respectively). To understand the relationship between the precorneal and prelens NIBUT values measured by this new objective and automated method, their correlation was also evaluated. No significant correlation was detected between the precorneal and prelens NIBUT values (for all; $p>0.05$ ).

A ROC curve was designed to determine the ability of the automated first and average NIBUT measurements to detect tear instability with respect to FBUT. The AUC values in the ROC curve were 0.842 (Cl: $0.731-0.953)$ for the precorneal first NIBUT and 0.810 (Cl: $0.684-0.935)$ for the precorneal average NIBUT prior to CL wear ( $p<0.001$; for both). The precorneal first and average NIBUT values prior to $C L$ wear had a good ability to determine tear instability (Fig. 2A). The AUC values were 0.586 (Cl: $0.404-$ $0.768)$ for the prelens first NIBUT and 0.619 (Cl: 0.442-0.796) for the prelens average NIBUT at the 30th minute of CL wear $(p=0.317$ and $p=0.166$, respectively). The prelens first and average NIBUT values after $\mathrm{CL}$ wear had not a reliable ability to detect tear instability (Fig. 2B). 
The ROC analysis suggested an ideal cut-off value of 8 seconds for the precorneal first NIBUT (maximum Youden index) with $78.1 \%$ sensitivity and $94.6 \%$ specificity in predicting tear instability. The ROC analysis suggested an ideal cut-off value of 12.65 seconds for the precorneal average NIBUT with $81.3 \%$ sensitivity and $77.8 \%$ specificity in predicting tear instability (Table 2). The ROC analysis did not recommend any reliable cut-off values for prelens NIBUT values.

Table 2

Receiver operating characteristics analysis of non-invasive tear break-up values capable of predicting tear instability

\begin{tabular}{|llllll|}
\hline Variable & $\begin{array}{l}\text { AUC } \\
(95 \% \mathrm{Cl})\end{array}$ & $\mathbf{p}$ & $\begin{array}{l}\text { Cut-off } \\
\text { Value }\end{array}$ & Se \% & Sp \% \\
\hline Precorneal first NIBUT & $\begin{array}{l}0.842 \\
(0.731-0.953)\end{array}$ & & & 78.1 & 94.6 \\
& 0.810 & $<0.001$ & 12.65 & 81.3 & 77.8 \\
\hline Precorneal average NIBUT & $(0.684-0.935)$ & & & & \\
& & & & & \\
AUC: Area Under Curve, Se: Sensitivity, Sp: Specificity, & & & & \\
NIBUT: Noninvasive Tear Break-Up Time & & & & \\
\hline
\end{tabular}

\section{Discussion}

This study examined the ability of the objective and automated precorneal and prelens NIBUT measurements to determine tear film instability in individuals using CLs. In this study, the previously internationally accepted FBUT cut-off value (less than 10 seconds as tear instability) was taken into consideration in identifying individuals with tear film instability $[11,14]$. We determined that the precorneal first and average NIBUT values automatically and objectively measured by the Sirius multifunctional topography device, were able to successfully detect tear instability. However, we found that the reliability and diagnostic ability of prelens first and average NIBUT measurements over CL was very low. As suggested in the literature, the reason for this may be that the device cannot distinguish between the thin areas and break-up areas in the prelens tear film since the prelens tear layer becomes very thin after $\mathrm{CL}$ wear [18]. Minor distortions caused by $\mathrm{CL}$ may also have limited the device's ability to reliably identify prelens tear film instability.

Bhandari et al. found a good correlation between FBUT and precorneal NIBUT in patients with dry eye and healthy individuals. They determined the precorneal NIBUT cut-off value as 6.2 seconds with $86.1 \%$ sensitivity and $81.1 \%$ specificity in the diagnosis of dry eye [13]. Best et al. found a significant correlation between FBUT and precorneal NIBUT before CL wear. They also stated that the basal NIBUT value could be used to predict CL drop-out, and those with a NIBUT value below $10 \mathrm{sec}$ should be followed up 
carefully [19]. Similarly, we found a significant correlation between FBUT and precorneal NIBUT in our study. In addition, we determined the optimum cut-off values for the precorneal first and average NIBUT measurements as 8 seconds and 12.65 seconds, respectively in determining tear instability with high specificity and sensitivity. We consider that studies with larger series are needed to provide more reliable cut-off values. We believe that future technological and software-related developments will reduce variations in the results of conducted studies.

In our study, we found no significant correlation between the prelens NIBUT values and the FBUT or precorneal NIBUT values, and the ROC analysis did not reveal a sufficiently reliable cut-off point for the prelens NIBUT values to predict tear instability. Similar to our study, Llorens-Quintana et al. reported that there was no significant correlation between precorneal NIBUT and prelens NIBUT [20]. Pult et al. and Chui et al. similarly noted that prelens NIBUT was not a reliable predictive method for identifying symptomatic and asymptomatic CL users [21, 22]. Glasson et al. found that the precorneal NIBUT value measured before $\mathrm{CL}$ wear showed a significant difference between the patients with and without $\mathrm{CL}$ intolerance, while the prelens NIBUT measured six hours after CL did not significantly differ between the two groups [5]. These results show that prelens NIBUT is not sufficient to determine CL intolerance. These studies support the results of our study in terms of demonstrating the low reliability of prelens NIBUT. Interestingly, we detected no correlation between precorneal and prelens NIBUT, which were both measured by the same automated method. We think that the identification of prelens tear kinetics by NIBUT is not as practical and easy as precorneal NIBUT due to the formation of different tear kinetics in the presence of lenses.

It has been reported in the literature that there is a relationship between the distribution and stability of tear film visual acuity [23]. Since NIBUT determines tear instability by evaluating topographic regularity indices, it also measures the anatomical function of the tear, as well as its physiological function. Since irregularities in the mires cause a decrease in image quality in the macula, it directly affects visual function $[1,24]$. This feature can help simultaneously evaluate both tear instability and its effect on vision quality using NIBUT in CL wearers with complaints about vision $[1,25]$. In our study, we determined that precorneal NIBUT was able to reliably detect tear instability. For this reason, we believe that this technique is useful in evaluating $\mathrm{CL}$ candidates with its potential to reveal more detail about the precorneal tear film that traditional clinical methods cannot detect.

To the best our knowledge, this is the first study to evaluate the ability of precorneal and prelens NIBUT to show tear stability with respect to FBUT using the Sirius multifunctional topography device. However, our results need to be confirmed by further studies with a larger sample size.

\section{Conclusion}

It was shown in our study that the precorneal NIBUT values measured automatically in the follow-up of $\mathrm{CL}$ users may be a fast, reliable, non-invasive and patient-friendly test based on a simple but effective imaging method in determining tear instability. In addition, this objective method may provide more 
detailed information than conventional methods about the tear film. However, we found no relationship between the prelens NIBUT values and the precorneal NIBUT or conventional FBUT values. For this reason, we think that prelens NIBUT offers only limited information about tear instability decision and it is not yet capable of adequately defining the dynamics of the tear film.

\section{Declarations}

\section{Compliance with ethical standards}

Ethical approval: All procedures performed in the studies involving human participants were in accordance with the ethical standards of the institutional and/or national research committee and with the 1964 Declaration of Helsinki and its later amendments or comparable ethical standards.

Data Availability: All authors declared that all data and materials as well as software application or custom code support their published claims and comply with field standards.

Consent to Participate (Ethics): Informed consent was obtained from all individual participants included in the study.

Consent to Publish (Ethics): Not applicable.

Clinical Trials Registration: Not applicable.

Authors' contributions: All authors contributed to the study conception and design. Material preparation, data collection and analysis were performed by [EM, MSD], [EM, MSD] and [EM, MSD]. The first draft of the manuscript was written by [EM] and all authors commented on previous versions of the manuscript. All authors read and approved the final manuscript.

Conflict of interest: The authors declare that they have no conflict of interest.

Funding: The authors declared that this study has received no financial support.

\section{References}

1. Craig JP, Willcox MDP, Argüeso P, Maissa C, Stahl U, Tomlinson A, Wang J, Yokoi N, Stapleton F; members of TFOS International Workshop on Contact Lens Discomfort. The TFOS international workshop on contact lens discomfort: Report of the contact lens interactions with the tear film subcommittee. Invest Ophthalmol Vis Sci 2013;54(11):TFOS:123-56.

2. Richdale K, Sinnott LT, Skadahl E, Nichols JJ. Frequency of and factors associated with contact lens dissatisfaction and discontinuation. Cornea 2007;26(2):168-74.

3. Gürdal C. Ocular Surface - Dry Eye and Contact Lens Relation. MN Oftalmol 2010;17(Suppl):60-4.

4. Aydın R, Özbek Söylemezoğlu Z, Durak İ. Comparison of Development of Dry Eye in Conventional Hydrogel and Silicone Hydrogel Contact Lens Users. Turk J Ophthalmol 2013;43(1):7-14. 
5. Glasson MJ, Stapleton F, Keay L, Willcox MD. The effect of short term contact lens wear on the tear film and ocular surface characteristics of tolerant and intolerant wearers. Cont Lens Anterior Eye. 2006;29(1):41-7.

6. Szczesna-Iskander DH, Iskander DR, Read SA, Alonso-Caneiro D. Noninvasive in vivo assessment of soft contact lens type on tear film surface quality. Invest Ophthalmol Vis Sci 2012;53(1):525-31.

7. Zhang J, Greenlee JD, Begley CG, Liu H, Simpson T, Chalmers RL, Begley CG, Liu H, Simpson T, Chalmers RL, Wu Z, Himebaugh NL, Jansen ME. A novel contact lens wettability grading scale related to changes in visual function. Invest Ophthalmol Vis Sci 2011;52(14): 6529.

8. Szczesna-Iskander DH, Alonso-Caneiro D, Iskander DR. Objective measures of pre-lens tear film dynamics versus visual responses. Optom Vis Sci 2016;93(8):872-880.

9. Smith J, Nichols KK, Baldwin EK. Current patterns in the use of diagnostic tests in dry eye evaluation. Cornea 2008;27(6):656-62.

10. Coşar B. Dry eye diagnosis methods. MN Oftalmoloji 2016;23(Suppl 1):9-14.

11. Wolffsohn JS, Arita R, Chalmers R, Djalilian A, Dogru M, Dumbleton K, Gupta PK, Karpecki P, Lazreg S, Pult H, Sullivan BD, Tomlinson A, Tong L, Villani E, Yoon KC, Jones L, Craig JP. TFOS DEWS II Diagnostic Methodology report. Ocul Surf 2017;15(3):539-74.

12. Gumus K, Crockett CH, Rao K, Yeu E, Weikert MP, Shirayama M, Hada S, Pflugfelder SC. Noninvasive assessment of tear stability with the tear stability analysis system in tear dysfunction patients. Invest Ophthalmol Vis Sci 2011;52(1):456-61.

13. Bhandari V, Reddy JK, Relekar K, Ingawale A, Shah N. Non-invasive assessment of tear film stability with a novel corneal topographer in Indian subjects. Int Ophthalmol 2016;36(6):781-90.

14. The definition and classification of dry eye disease: report of the Definition and Classification Subcommittee of the International Dry Eye WorkShop (2007). Ocul Surf 2007;5(2):75-92.

15. Metz CE. Basic principles of ROC analysis. Seminars in nuclear medicine. 1978;8(4):283-98.

16. Bewick V, Cheek L, Ball J. Statistics review 13: receiver operating characteristic curves. Critical care (London, England). 2004;8(6):508-12.

17. Bantis LE, Nakas CT, Reiser B. Construction of confidence regions in the ROC space after the estimation of the optimal Youden index-based cut-off point. Biometrics. 2014;70(1):212-23.

18. Guillon M, Dumbleton KA, Theodoratos P, Wong S, Patel K, Banks G, Patel T. Association between contact lens discomfort and pre-lens tear film kinetics. Optom Vis Sci 2016;93(8):881-91.

19. Best N, Drury L, Wolffsohn JS. Predicting success with silicone-hydrogel contact lenses in new wearers. Cont Lens Anterior Eye 2013;36(5):232-7.

20. Llorens-Quintana C, Mousavi M, Szczesna-Iskander D, Iskander DR. Non-invasive pre-lens tear film assessment with high-speed videokeratoscopy. Cont Lens Anterior Eye 2018;41(1):18-22.

21. Pult $H$, Purslow $C$, Berry M, Murphy PJ. Clinical tests for successful contact lens wear: relationship and predictive potential. Optom Vis Sci 2008;85(10):924-9. 
22. Chui WS, Cho P, Brown B. Soft contact lens wear in Hong Kong-Chinese: predicting success. Ophthalmic Physiol Opt 2000;20(6):480-6.

23. Kolbe 0 , Zimmermann F, Marx S, Sickenberger W. Introducing a novel in vivo method to access visual performance during dewetting process of contact lens surface. Cont Lens Anterior Eye 2020;43(4):359-65.

24. Goto E, Ishida R, Kaido M Dogru M, Matsumoto Y, Kojima T, Tsubota K. Optical aberrations and visual disturbances associated with dry eye. Ocul Surf 2006;4(4):207-13.

25. Thai LC, Tomlinson A, Ridder WH. Contact lens drying and visual performance: the vision cycle with contact lenses. Optom Vis Sci 2002;79(6):381-8.

\section{Figures}
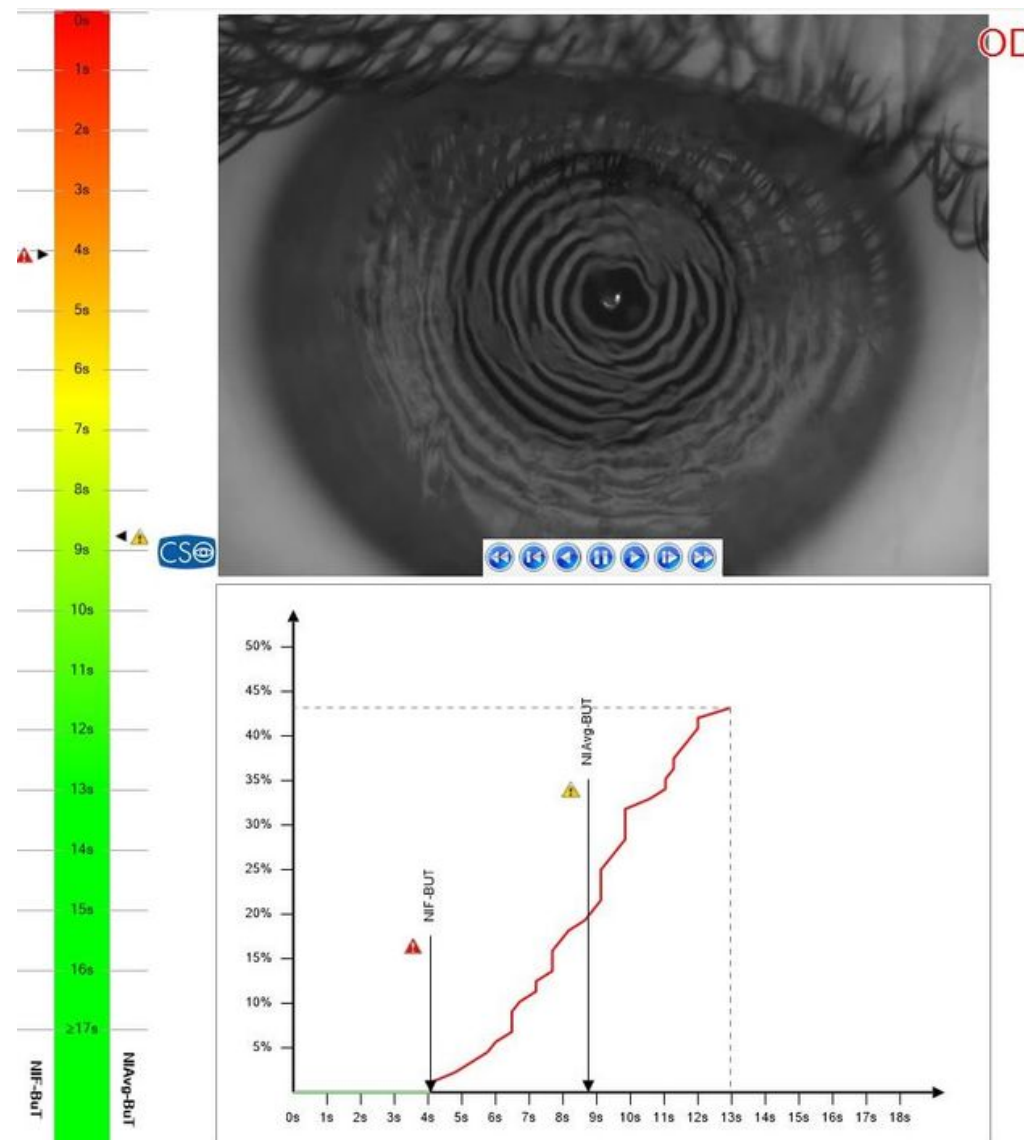
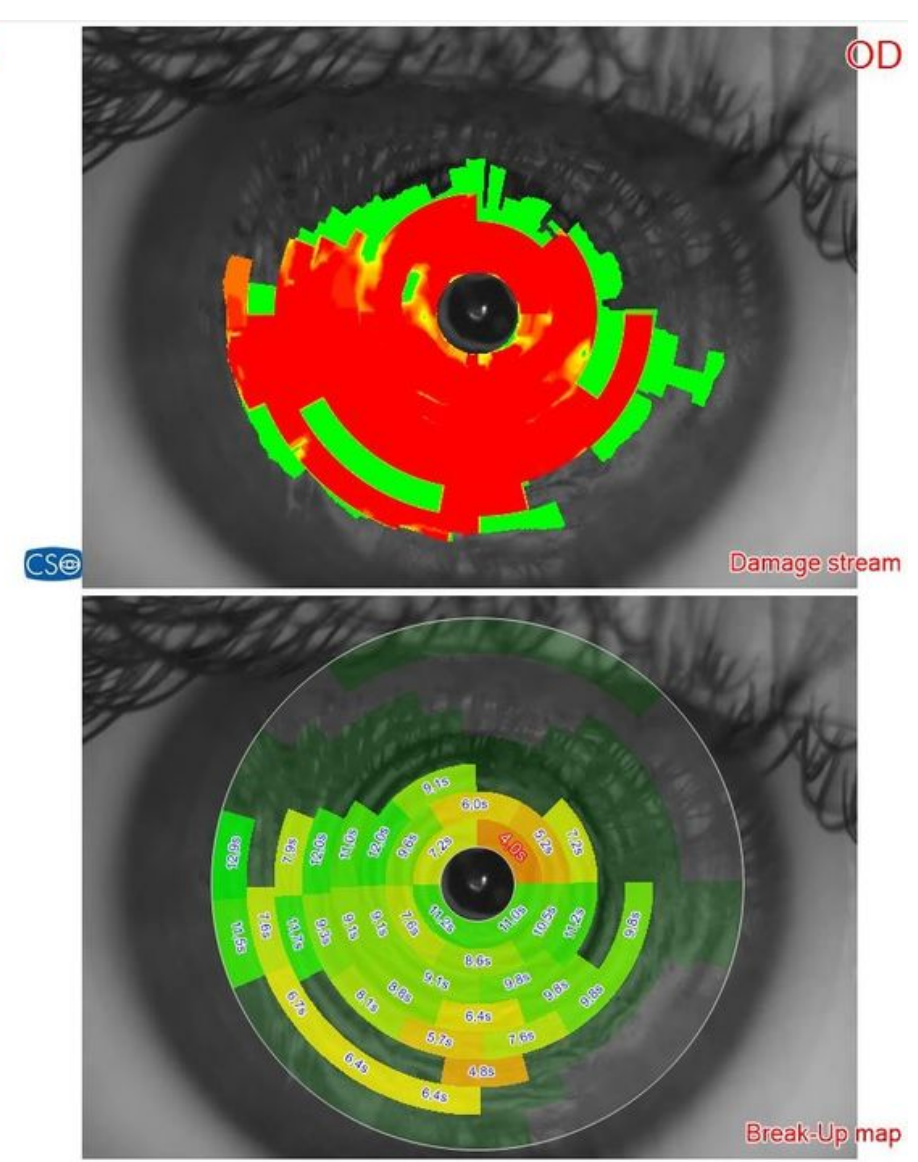

\section{Figure 1}

The non-invasive tear break up time (NIBUT) maps of one of the participants in our study. (The results are given as a real-time videokeratoscopy recording in which a dynamic map of the break-up flow is shown in the upper left, and the number and time of the breakpoints that occur in real time in the upper right of the figure. Furthermore, the bottom left of the figure gives the percentage of tears that are broken depending on the time during the measurement, and the bottom right presents a graphical map showing first and average NIBUTs, where the break-up times in different areas are shown numerically.) 

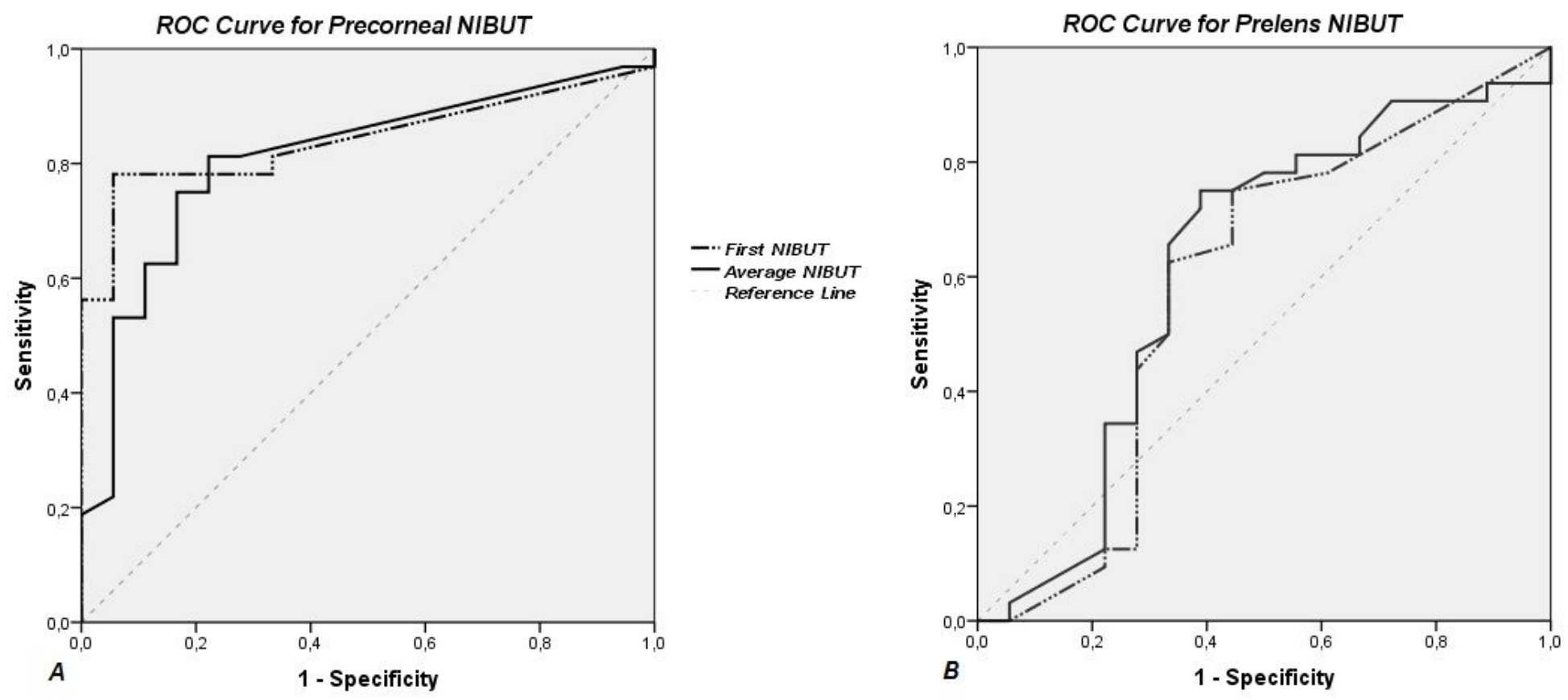

Figure 2

Receiver operator characteristic curve curves showing the performance of first and average non-invasive tear break up time (NIBUT) parameters for determining tear instability using Sirius multifunctional topography. [The area under curve were $0.842(p<0.001)$ for the precorneal first NIBUT and 0.810 $(p<0.001)$ for the precorneal average NIBUT prior to CL wear $(A)$. The AUC values were $0.586(p=0.317)$ for the prelens first NIBUT and $0.619(p=0.166)$ for the prelens average NIBUT at the 30th minute of CL wear (B).] 